\title{
Determination of a non-measurable quantity using information from calculations and experimental measurements: application to the damage rate determination
}

\author{
Stéphane Bourganel ${ }^{1, a}$, and Jean-Claude Nimal ${ }^{1}$ \\ ${ }^{1}$ CEA/Saclay - DEN/DANS/DM2S/SERMA - Bâtiment 470, PC 57 - 91191 Gif sur Yvette Cedex - France
}

\begin{abstract}
This article presents a method dedicated to the determination of the best value of a required quantity which is estimated by calculation, using information closely related, obtained by measurements and calculations. This best value, called thereafter the "target", is not measurable in most cases. DPA and high energy neutron fluence (typically higher than $1 \mathrm{MeV}$ ) involved in vessel surveillance programs, using measurements of dosimeters, are some examples of application of this methodology. This methodology is applied without spectrum adjustment, but the spectrum shape is implicitly taken into account.

In this article, an example is presented based on the FLUOLE-2 experimental program, which is developed and conducted by CEA. Neutron information is derived from a set of different kinds of neutron dosimeters. The objective is to estimate the best value of reaction rate values for each kind of dosimeters. All calculations are carried out using TRIPOLI-4 3D pointwise Monte Carlo code, and DARWIN/PEPIN2 depletion code.
\end{abstract}

This article presents a method dedicated to the determination of the best value of a required quantity which is estimated by calculation, using information closely related, obtained by measurements and calculations. This best value, called thereafter the "target", is not measurable in most cases. DPA and high energy neutron fluence (typically higher than $1 \mathrm{MeV}$ ) involved in vessel surveillance programs, using measurements of dosimeters, are some examples for which this methodology can be applied. In this methodology, spectrum adjustment is not used, but the spectrum shape is implicitly taken into account.

\section{General case}

Let $C_{k}$ and $M_{k}$ be a calculated and a measured value of the physical quantity $\mathrm{k}$ respectively (a reaction rate for instance; $\mathrm{k}$ spans 1 to $\mathrm{NK}$ ), and $\mathrm{C}_{0}$ an estimate of the target value also obtained by calculation. $\mathrm{C}_{0}$ is a physical quantity close to $\mathrm{C}_{\mathrm{k}}$. First, $\mathrm{C}_{0}$ may be enhanced, using each of the NK measured and calculated information $<\mathrm{C}_{\mathrm{k}}, \mathrm{M}_{\mathrm{k}}>$, providing NK improved values called observances, what will be noted $R_{k}$ further. These values represent imperfect results of the target value deduced from each dosimeter. These $R_{k}$ values can be linearly combined to determine the best estimate target value $\mathrm{R}_{0} . \mathrm{R}_{0}$ represents an improvement of the first estimated target value $\mathrm{C}_{0}$. Indeed, $\mathrm{C}_{0}$ is degraded by imperfections in the modelling (lack of knowledge or uncertainties on modelling data, cross

\footnotetext{
${ }^{\mathrm{a}}$ Corresponding author: sbourganel@cea.fr
}

sections ...). By contrast, measurements are perfect with regard to the reality, even if they are biased by experimental uncertainties.

The determination of the $\mathrm{R}_{0}$ value requires a weighting of the contribution of each observance $R_{k}$. To achieve this goal, two kinds of elements are used:

- The concept of proximity between each couple $<\mathrm{C}_{\mathrm{k}}$, $\mathrm{M}_{\mathrm{k}}>$ and the imperfect target $\mathrm{C}_{0}$, which incorporates the representativeness of the couples $<\mathrm{C}_{\mathrm{k}}, \mathrm{M}_{\mathrm{k}}>$ with regard to the target.

- The uncertainty associated to each calculated and measured values $\left(<\mathrm{C}_{\mathrm{k}}, \mathrm{M}_{\mathrm{k}}>\right)$.

\section{Application of the methodology: neutron flux $>1 \mathrm{MeV}$ calculation}

Some details to carry out a numerical application of the methodology described above, is presented in this section. The aim is to determine a damage rate, based on neutron flux with energy greater than $1 \mathrm{MeV}: \phi_{1}$. Here, this high energy neutron flux is the required target value. Required information comes from measurements and calculations which are linked to a set of NK neutron dosimeters:

- $\quad M_{k}$ : The reaction rate values which are deduced from the measured dosimeter activities. $\mathrm{M}_{\mathrm{k}}$ values are strongly linked to the reality of the irradiation. They are also independent of the system modelling.

- $C_{k}$ : The calculated reaction rate values using 
TRIPOLI-4 Monte Carlo code [1].

- $\phi_{1, T 4}$ : The neutron flux with energy greater than 1 $\mathrm{MeV}$, also calculated with TRIPOLI- $4^{\circledR}$ code. $\phi_{1, T 4}$ (called $\mathrm{C}_{0}$ in the previous section) represents an estimate of the target value.

NK observances $\phi_{1, k}$ (called $\mathrm{R}_{\mathrm{k}}$ in the previous section) are defined, representing NK values of the neutron flux with energy greater than $1 \mathrm{MeV}$ which are "seen" by dosimeters. Each of these observances is deduced by measurement and calculation. Impact of measurements is taken into account in the $\phi_{1, k}$ determination in the following manner: the reaction rate values $M_{k}$ deduced from measurements are divided by the calculated crosssection value $>1 \mathrm{MeV}\left(\sigma_{k, 1} \mathrm{MeV}\right)$ as shown in the formula (1). This cross-section value is the ratio between the calculated reaction rate $C_{k}$, and the calculated flux $>1$ $\mathrm{MeV}$ value $\phi_{1, T 4} \cdot \sigma_{k, 1 \mathrm{MeV}}$ is therefore independent of the power level of the system modelling.

$$
\phi_{1, k}=\frac{M_{k}}{\sigma_{k, 1} \mathrm{MeV}}=\frac{M_{k}}{C_{k}} \times \phi_{1, T 4}
$$

Formula (1) is valid only if dosimeters were irradiated with a single uniform cycle. This formula must be changed when several cycles are considered [2].

Besides this methodology is applied to the determination the neutron fluence, it can be generalized to others physical quantities as DPA.

The best estimate target value $\phi_{1, \text { estimate }}$ is obtained by linear combination of the NK observances $\phi_{1, k}$ :

$$
\phi_{1, \text { estimate }}=\sum_{k=1}^{N K} W_{k} \times \phi_{1, k}
$$

The importance of the observance $\phi_{1, k}$ in the determination of the target value $\phi_{1, \text { estimate }}$ is quantified by the weight value $W_{k}$. This weight is obtained by minimizing the variance associated to the best target value $\phi_{1, \text { estimate }}$. The best estimate of the target value $\phi_{1, e s t i m a t e}$ and its associated variance value $\sigma^{2}\left(\phi_{1, \text { estimate }}\right)$ are given in formulae (3) and (4).

$$
\begin{gathered}
\phi_{1, \text { estimate }}=\frac{\overrightarrow{1^{t}} \times\left\|C V \phi_{1}\right\|^{-1} \times \overrightarrow{\phi_{1}}}{\overrightarrow{1^{t}} \times\left\|C V \phi_{1}\right\|^{-1} \times \overrightarrow{1}} \\
=\sum_{k=1}^{N K} W_{k} \times \phi_{1, k}
\end{gathered}
$$

(4)

$$
\begin{aligned}
& \sigma^{2}\left(\phi_{1, \text { estimate }}\right) \\
& \quad=\frac{1}{\overrightarrow{1^{t}} \times\left\|C V \phi_{1}\right\|^{-1} \times \overrightarrow{1}}
\end{aligned}
$$

In formulae (3) and (4), $\left\|C V \phi_{1}\right\|$ is the variancecovariance matrix associated to the NK observances $\phi_{1, k}$. It is a square matrix of order NK. By definition, this matrix is positive-definite and thus, it is invertible. $\overrightarrow{\phi_{1}}$ is the $\phi_{1, k}$ vector of NK observances.

This matrix is obtained by the simple matrix expression for error propagation:

$$
\left\|C V \phi_{1}\right\|=\|S\|^{t} \times\left\|C V_{n i v 3}\right\| \times\|S\|
$$

Formula (5) is based on a linear approximation. $\|S\|$ is a sensitivity matrix which is easily deduced from formula (1).

$\left\|C V_{\text {niv3 }}\right\|$ is a square matrix of order $(2 \times N K+1)$. It represents the variance-covariance matrix of the $\mathrm{NK}$ measured values $\mathrm{M}_{\mathrm{k}}$ and the $(\mathrm{NK}+1)$ calculated values $\mathrm{C}_{\mathrm{k}}$. It represents uncertainties associated to measured values, modelling data, nuclear data, etc. Paragraph 3 is dedicated to the determination of the $\left\|\mathrm{CV}_{\text {niv3 }}\right\|$ matrix.

\section{3 determination of the $\left\|C V_{\text {niv3 }}\right\|$ matrix}

The $\left\|\mathrm{CV}_{\text {niv3 }}\right\|$ matrix comprises 3sub-matrices:

- One square sub-matrix of order NK, representing uncertainties on measured values. These sub-matrix elements are given by experimenters.

- One square sub-matrix of order $(\mathrm{NK}+1)$, representing variance and covariance values associated to calculated values.

- Two rectangular sub-matrices $\mathrm{NK} \times(\mathrm{NK}+1)$ representing correlations between measured and calculated values. We should note that these two kinds of values are usually uncorrelated.

This paragraph is dedicated to the determination of sub-matrix representing calculated values. This is the most difficult point to deal with the problem of uncertainties. Each element of this sub-matrix is given by formula (6).

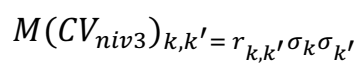

$r_{k k^{\prime}}$ is the correlation coefficient between dosimeters $k$ and $k^{\prime} . k$ is between 0 an NK. $k=0$ corresponds to the calculated target value, and $1 \leq \mathrm{k} \leq \mathrm{NK}$ to calculated reaction rate values associated to dosimeters. Data for completing the $\left\|C V_{\text {niv3 }}\right\|$ matrix may have different origins:

- A methodologically uncertainty propagation method. In this way, variance and covariance values are rigorously linked to uncertainties associated with the problem (technological data, nuclear data...). $r_{k, k}$, values are then known. The URANIE platform [3] developed by CEA is one example to address this need. 
- $\quad$ Results from ESTIMA computer program when only variance values are available. ESTIMA allows calculating covariance values according to a methodology presented in chapter 4. Variance values are known through feedback. A practical application of this way is presented in chapter 5 where $r_{k, k}$, values are deduced from neutron spectra.

- Results from CORRELA computer program (see chapter 4) when reliable uncertainty data are not available. $\mathrm{r}_{\mathrm{k}, \mathrm{k}}$, values are also deduced from neutron spectra.

\section{ESTIMA computer program}

A computer program has been developed to carry out numerical applications. It consists of a main program called ESTIMA, dedicated to matrix calculation. It can be used for any kinds of problems. It comprises a function named CORRELA, dedicated to the calculation of the $r_{k, k^{\prime}}$ correlation factors. ESTIMA allows obtaining the best target value $\phi_{1 \text {,estimate, and the associated }}$ variance $\sigma^{2}\left(\phi_{1}\right)$. This package allows to process a set of covariance matrices that might be generated randomly.

\section{Numerical applications}

Numerical applications are presented in this paper. They are based on FLUOLE-2 program [4], and concern reaction rate calculations.

\subsection{FLUOLE-2 program}

FLUOLE-2 [4] is a benchmark-type experiment dedicated to neutron attenuation analysis with the aim of improving the TRIPOLI- ${ }^{\circledR}$ [1] Monte Carlo code validation. This two-year program has been developed by CEA (Commissariat à l'Energie Atomique et aux Energies Alternatives), with the financial support of EDF, to be representative of 900 and 1450 MWe Pressurized Water Reactors. In this perspective, different stainless steel structures have been designed and appropriately positioned inside the EOLE facility [5]. In FLUOLE-2 program, a set of dosimeters were irradiated and the activity was measured. Calculated activity of each dosimeter was obtained with a specific calculation scheme based on TRIPOLI- $4{ }^{\circledR}$ Monte Carlo code, and DARWIN/PEPIN2 depletion code.

\subsection{TRIPOLI-4 ${ }^{\circledR}$}

TRIPOLI- $4^{\circledR}[1]$ is a 3D transport code using full pointwise cross section data. It is dedicated to radiation protection and shielding, nuclear criticality safety, fission and fusion reactor design, and nuclear instrumentation. It is used as a reference tool by CEA, EDF, and several other industrial or institutional partners. In this study, TRIPOLI- $4{ }^{\circledR}$ is used to calculate the neutron source distribution in the core, the neutron propagation through the different structures, and the fission rate values inside fission chambers.

\subsection{DARWIN/PEPIN2}

DARWIN/PEPIN2 [6] solves Bateman's generalized differential equations governing the time dependence of isotope concentrations. This code may be coupled with the TRIPOLI- $4{ }^{\circledR}$ code. In this analysis, the DARWIN/PEPIN2 code is dedicated to dosimeter activation calculations.

\subsection{Applications: general}

For the numerical application, 8 dosimeters have been selected from the FLUOLE-2 analysis result database. These dosimeters were irradiated in the same position from the core [4]. The analysis of 8 dosimeters shows relatively good agreement between calculated and measured activity values, as presented in Table 1 . Average $\mathrm{C} / \mathrm{M}$ values for all dosimeters grouped by type are similar to those results.

Table 1. C/M results from FLUOLE-2 analysis

\begin{tabular}{|c|c|}
\hline Dosimeters & $\mathrm{C} / \mathrm{M}$ \\
\hline${ }^{27} \mathrm{Al}(\mathrm{n}, \alpha)$ & $1.00 \pm 0.03$ \\
\hline${ }^{54} \mathrm{Fe}(\mathrm{n}, \mathrm{p})$ & $1.00 \pm 0.03$ \\
\hline${ }^{115} \mathrm{In}\left(\mathrm{n}, \mathrm{n}^{\prime}\right)$ & $0.98 \pm 0.03$ \\
\hline${ }^{58} \mathrm{Ni}(\mathrm{n}, \mathrm{p})$ & $1.00 \pm 0.03$ \\
\hline${ }^{103} \mathrm{Rh}\left(\mathrm{n}, \mathrm{n}^{\prime}\right)$ & $0.86 \pm 0.05$ \\
\hline${ }^{117} \mathrm{Sn}(\mathrm{n}, \mathrm{n})$ & $1.05 \pm 0.03$ \\
\hline${ }^{46} \mathrm{Ti}(\mathrm{n}, \mathrm{p})$ & $1.00 \pm 0.03$ \\
\hline${ }^{51} \mathrm{~V}(\mathrm{n}, \alpha)$ & $0.98 \pm 0.04$ \\
\hline
\end{tabular}

Numerical applications have the objective to show that it is possible to enhance the target value using sometimes unsatisfying results, and running ESTIMA program with calculated and measured results. This improvement consists of a significant reduction of the variance associated to the target value. The $\left\|\mathrm{CV}_{\text {niv3 }}\right\|$ matrix is filled as described in chapter 3: experimental uncertainties are given by measurement laboratory, and calculation uncertainties are derived through feedback on PWR uncertainty analysis.

Dosimeters can be divided into two categories:

- Dosimeters with poor $\mathrm{C} / \mathrm{M}$ ratio. These results may be due to inadequate knowledges of modelling data (nuclear data, technological data ...) or incorrect measurement. Rhodium and tin dosimeters are part of this category.

- Dosimeters with very good $\mathrm{C} / \mathrm{M}$ ratio. All others dosimeters are classified in this group.

Further on, the main reaction rate for nickel, rhodium, and tin dosimeters will be considered as target values.

\subsection{Numerical applications}

\subsubsection{General}

Numerical applications have the objective to demonstrate the robustness of the methodology proposed in the paper. The possibility to improve target values is shown, 
including when data involved in the analysis process $(\mathrm{C} / \mathrm{M}$ calculations) are imperfect.

In our test, we will assume that uncertainty on the neutron spectrum and reaction rates for each dosimeter, both calculated with TRIPOLI- $4^{\circledR}$, is altered by a lack of knowledge of nuclear data. The impact of this on results given by ESTIMA will be checked. To achieve this goal, the calculated spectrum is deliberately distorted by the use of a set of multiplying factors. For each case, the target value is then calculated as well as the associated uncertainty, called residual uncertainty.

\subsubsection{Altering spectrum}

The calculated spectrum is altered using a multiplying factor $\mathrm{F}_{\mathrm{p}}(\mathrm{E})$ defined as follows:

- $0.04 \mathrm{MeV} \leq \mathrm{E} \leq 8 \mathrm{MeV}: \mathrm{F}_{\mathrm{p}}(\mathrm{E})$ increases linearly, with $\mathrm{F}_{\mathrm{p}}(0.04 \mathrm{MeV})=\mathrm{a}_{\mathrm{p}}$ and $\mathrm{F}_{\mathrm{p}}(8 \mathrm{MeV})=1$.

- $8 \mathrm{MeV} \leq \mathrm{E} \leq 20 \mathrm{MeV}: \mathrm{F}_{\mathrm{p}}(\mathrm{E})$ increases linearly, with $\mathrm{F}_{\mathrm{p}}(8 \mathrm{MeV})=1$ and $\mathrm{F}_{\mathrm{p}}(20 \mathrm{MeV})=1 / \mathrm{a}_{\mathrm{p}}$.

The $a_{p}$ factor values will be specified further. Beside this example of a voluntary degradation of the spectrum, other combinations are possible.

\subsubsection{Application \#1: nickel dosimeter}

In this test, ${ }^{58} \mathrm{Ni}(\mathrm{n}, \mathrm{p})$ reaction rate is the target value. A high level of confidence on calculated and measured values associated to this reaction is provided by a very good agreement between these two values for this dosimeter $(\mathrm{C} / \mathrm{M}=1.00 \pm 0.03)$. Besides, this reaction in deemed to be a reference reaction in the field of dosimetry nuclear data.

Impact of altering spectra on the target value (reaction rate) given by ESTIMA is gathered in Table 2: first, $\mathrm{C} / \mathrm{M}$ ratios considering altering spectra are calculated, then, $\mathrm{C} / \mathrm{M}$ ratios from ESTIMA are shown.

Table 2. Application \#1: C/M ratios for nickel dosimeter

\begin{tabular}{|c|c|c|}
\hline $\mathrm{a}_{\mathrm{p}}$ factors & $\begin{array}{c}\text { Results from } \\
\text { altering spectra }\end{array}$ & $\begin{array}{c}\text { Results from } \\
\text { ESTIMA }\end{array}$ \\
\hline 0.75 & 0.865 & 1.004 \\
\hline 0.80 & 0.892 & 1.005 \\
\hline 0.90 & 0.947 & 1.005 \\
\hline 0.95 & 0.974 & 1.005 \\
\hline 1.00 & 1.001 & 1.005 \\
\hline 1.05 & 1.028 & 1.006 \\
\hline 1.10 & 1.056 & 1.006 \\
\hline 1.20 & 1.110 & 1.006 \\
\hline 1.25 & 1.137 & 1.007 \\
\hline
\end{tabular}

ESTIMA provides stable $\mathrm{C} / \mathrm{M}$ values, close to the FLUOLE-2 reference result (Table 1), whereas the altering spectra produce erroneous reaction rates heavily dependent on the altering factors $\mathrm{a}_{\mathrm{p}}$.

\subsubsection{Application \#2: rhodium dosimeter}

In this application, ${ }^{103} \mathrm{Rh}(\mathrm{n}, \mathrm{n}$ ') reaction rate is the target value. Measured results for rhodium dosimeters are subject to some uncertainties: X-rays (around $20 \mathrm{keV}$ ) emitted by ${ }^{103 \mathrm{~m}} \mathrm{Rh}$ are largely absorbed by the dosimeter itself though it is very thin $(50 \mu \mathrm{m})$. This involves significant corrective factors. Furthermore, the X-rays emission probabilities are known with an uncertainty of $7 \%$.

As in application \#1, Table 3 shows impact of the perturbation of the altering spectra, and the results of ESTIMA

Table 3. Application \#2: C/M ratios for rhodium dosimeter.

\begin{tabular}{|c|c|c|}
\hline $\mathrm{a}_{\mathrm{p}}$ factors & $\begin{array}{c}\text { Results from } \\
\text { altering spectra }\end{array}$ & $\begin{array}{c}\text { Results from } \\
\text { ESTIMA }\end{array}$ \\
\hline 0.75 & 0.795 & 0.908 \\
\hline 0.80 & 0.808 & 0.900 \\
\hline 0.90 & 0.833 & 0.886 \\
\hline 0.95 & 0.846 & 0.887 \\
\hline 1.00 & 0.859 & 0.874 \\
\hline 1.05 & 0.872 & 0.869 \\
\hline 1.10 & 0.885 & 0.863 \\
\hline 1.20 & 0.910 & 0.853 \\
\hline 1.25 & 0.923 & 0.850 \\
\hline
\end{tabular}

As in the previous case, ESTIMA provides stable C/M results. This trend confirms that the measured reaction rate value needs to be revised.

\subsubsection{Application \#3: tin dosimeter}

In this third application, ${ }^{117} \mathrm{Sn}(\mathrm{n}, \mathrm{n}$ ') reaction rate is the target value. It should be noted that the use of tin dosimeters is fairly new, and the inelastic scattering cross section for ${ }^{117} \mathrm{Sn}$ is probably not well known. Indeed, depending on the origin of nuclear data (JEFF3.1.1, Endf/B-VII ...), the calculated reaction rate can vary by twice as much. In addition, this reaction is available neither in the International Reactor Dosimetry File (IRDF2002) nor in the new Dosimetry library IRDFF. For the FLUOLE-2 analysis, inelastic scattering cross section data for ${ }^{117} \operatorname{Sn}(n, n$ ') reaction come from the JENDL/A-96 nuclear library which performs a little better than other libraries. By contrast, the activity measurement doesn't have a particular technical difficulty.

As in applications \#1 and \#2, Table 4 shows impact of altering spectra on $\mathrm{C} / \mathrm{M}$ results obtained from altering spectrum, and using ESTIMA.

Table 4. Application \#2: $\mathrm{C} / \mathrm{M}$ ratios for tin dosimeter.

\begin{tabular}{|c|c|c|}
\hline $\mathrm{a}_{\mathrm{p}}$ factors & $\begin{array}{c}\text { Results from } \\
\text { altering spectra }\end{array}$ & $\begin{array}{c}\text { Results from } \\
\text { ESTIMA }\end{array}$ \\
\hline 0.75 & 0.939 & 1.029 \\
\hline 0.80 & 0.962 & 1.038 \\
\hline 0.90 & 1.003 & 1.052 \\
\hline 0.95 & 1.032 & 1.058 \\
\hline 1.00 & 1.055 & 1.063 \\
\hline
\end{tabular}




\begin{tabular}{|l|l|l|}
\hline 1.05 & 1.078 & 1.067 \\
\hline 1.10 & 1.101 & 1.071 \\
\hline 1.20 & 1.147 & 1.077 \\
\hline 1.25 & 1.170 & 1.080 \\
\hline
\end{tabular}

ESTIMA provides, once again, stable values. The nuclear data problem is confirmed by this result.

\section{Conclusion}

In light of the above considerations, an estimate of uncertainties associated to calculated reaction rate results can be given. It is the same with uncertainties associated to measured reaction rate values even if they are usually given by the measurement laboratories. Rhodium dosimeter is an exception: an uncertainty of $13 \%$ for measurement seems to be more adequate. For calculated results, uncertainty is obtained by CORRELA, except for tin dosimeter. For that kind of dosimeter, cross section data seem to be incorrect because $\mathrm{C} / \mathrm{M}$ ratio is not perfect, and results from ESTIMA remain unchanged, regardless of the altering spectrum.

The methodology presented in this article allows to make up for a lack of information to determinate and to enhance a requested result, using an appropriate weighting of available information (typically calculation and/or measure data). This methodology also alerts when any incorrect data are used in a calculation. Although a good neutron flux is provided by ESTIMA, agreement between measurement and calculation cannot be achieved when external data are incorrect (measurement results or cross section data for instance).

The authors would like to thank Mr. Jean-Marc Martinez (CEA/Saclay) for many fruitful discussions [6].

\section{References}

1. "TRIPOLI- $4{ }^{\circledR}$ version $8.1,3 \mathrm{D}$ general purpose continuous energy Monte Carlo Transport code", NEA-1716 TRIPOLI-4 VERS. 8.1, https://www.oecd-

nea.org/tools/abstract/detail/nea-1716

2. Bevilacqua, R. Lloret, J.C. Nimal, S.H. Zengh, C. Rieg, "Special dosimetry at Saint Laurent B1A", ASTM-EURATOM 1993.

3. F. Gaudier, "URANIE : The CEA/DEN Uncertainty and Sensitivity platform", Procedia Social and Behavioral Sciences 2 (2010) 76607661

4. N. Thiollay et al, "FLUOLE-2: an Experiment for PWR Pressure Vessel Surveillance", Fifteenth International Symposium on Reactor Dosimetry (ISRD 15), Aix-en-Provence, France, May 18-23, 2014.

5. JC. Bosq, M. Antony, J. Di-Salvo, J.C. Klein, N. Thiollay, P. Blaise, P. Leconte, "The use of EOLE and MINERVE critical facilities for the
Generation 3 light water reactors studies", Proc. Of ICAPP 2011, Nice, France, May 2-5, 2011

6. A. Tsilanizara et al, "Experimental validation of the DARWIN2.3 package for fuel cycle applications", PHYSOR 2012, Knoxville, Tennessee, USA, April 15-20, 2012.

7. J.M. Martinez (CEA/Saclay), Private communication, 2014 September $15^{\text {th }}$. 